\title{
Investigation of solvability conditions of certain boundary value problem by perturbation method
}

\author{
Akram Hassan Mahmood \\ Department of Mathematics / College of Education \\ University of Mosul
}

Received

$18 / 11 / 2008$
Accepted

16 / 02 / 2009

المالخص:

مم في هذا البحث درلسةشروط الطل لمعادلة تفاضلية جزئية معينة ذاتشروط حدوية متجلنة تحتوي معلمة صغيرة. هذه المسألة حلت بلستخدلم لسٔلوب التشوبيث. هنا تعلملنا مع المعادلة الفاضلية الجزئية

$\varphi_{x x}+\varphi_{y y}+w^{2} \varphi=0$

مع للثروط الحدونية

$\varphi_{y}(x, a)-\varphi_{y}(x, b)=0$

$\left.\varphi(x, y)\right|_{y=a+\varepsilon \sin k_{w^{x}}}-\left.\varphi(x, y)\right|_{y=b+\varepsilon \sin k_{w^{x}}}=0$

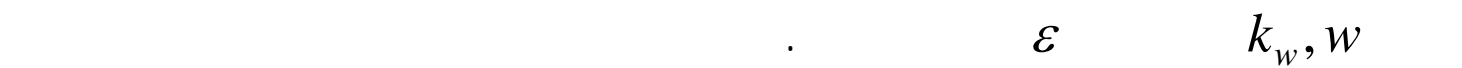
القليلة الأوله لتبسط التثوبيث.

\section{Abstract}

In this paper we study the solvability conditions under which certain partial differential equation with homogenous boundary conditions containing small parameter has a solution by using perturbation technique.

Here we deal with the following partial differential equation

$\varphi_{x x}+\varphi_{y y}+w^{2} \varphi=0$

with boundary conditions

$\varphi_{y}(x, a)-\varphi_{y}(x, b)=0$

and 
$\left.\varphi(x, y)\right|_{y=a+\varepsilon \sin k_{w^{x}}}-\left.\varphi(x, y)\right|_{y=b+\varepsilon \sin k_{w^{x}}}=0$

where $k_{w}, w$ are constants and $\varepsilon$ is small parameter.

According to this method, the solution of the problem is represented by the first few terms of a perturbation expansion.

\section{Introduction}

Many of the problems faced to day by physicists, engineers and applied mathematicians involve difficulties, such as nonlinear boundary conditions at complex known or unknown boundaries can be solved by approximation methods. One of them is perturbation method, according to these techniques, the solution of the problem is represented by the first few terms of a perturbation expansion [5], perturbation methods have been used [2] for solving elliptic equations with small nonlinearity. In [4] the author used the perturbation method which is given in [6] to find the solvability conditions for certain eigenvalue problem of fourth order. In [3] various perturbation problem are arise in the theory of lubrication.

Homotopy perturbation method is applied for solving fourth order boundary value problems by [7].

In this study we give a generalization of the boundary conditions that were given by [1]. Here we deal with the following partial differential equation

$\varphi_{x x}+\varphi_{y y}+w^{2} \varphi=0$

with boundary conditions

$\varphi_{y}(x, a)-\varphi_{y}(x, b)=0$

$\left.\varphi(x, y)\right|_{y=a+\varepsilon \sin k_{w} x}-\left.\varphi(x, y)\right|_{y=b+\varepsilon \sin k_{w^{x}}}=0$

where $k_{w}, w$ are constants and $\varepsilon$ is small parameter.

\section{Solvability conditions for partial differential equations with boundary conditions}

The study is divided as the following steps:

\section{I - Transfer the boundary conditions}

Transfer the boundary conditions from

$y=a+\varepsilon \sin k_{w} x, y=b+\varepsilon \sin k_{w} x$ to $y=a, y=b \quad$ respectively

Let $\varphi=\varphi_{0}(x, y)+\varepsilon \varphi_{1}(x, y)+\varepsilon^{2} \varphi_{2}(x, y)+\ldots .$.

We note that the boundary condition (1.3) is imposed at $y=a+\varepsilon \sin k_{w} x, y=b+\varepsilon \sin k_{w} x$, and hence $\varepsilon$ appears in the argument of $\varphi$ as well as in the coefficients.

Since the usual procedure in perturbation methods is to equate coefficients of equal powers of $\varepsilon$, we will not be able to do that unless $\varepsilon$ 
is removable from the argument. To do this, we transfer the boundary conditions from

$y=a+\varepsilon \sin k_{w} x \quad$ to $y=a, y=b+\varepsilon \sin k_{w} x$ to $y=b$

by a Taylor series expansion.

We write $\varphi(x, y)$ at $y=a+\varepsilon \sin k_{w} x, y=b+\varepsilon \sin k_{w} x$ and expanding in a Taylor series about $y=a, y=b$, we have $\varphi\left(x, a+\varepsilon \sin k_{w} x\right)=\varphi(x, a)+\varphi_{y}(x, a) \varepsilon \sin k_{w} x+\frac{1}{2 !} \varphi_{y y}(x, a) \varepsilon^{2} \sin ^{2} k_{w} x+\ldots \ldots$ $\varphi\left(x, b+\varepsilon \sin k_{w} x\right)=\varphi(x, b)+\varphi_{y}(x, b) \varepsilon \sin k_{w} x+\frac{1}{2 !} \varphi_{y y}(x, b) \varepsilon^{2} \sin ^{2} k_{w} x+\ldots \ldots$

Substituting these Taylor series expansions into (1.3), we obtain $\varphi(x, a)+\varphi_{y}(x, a) \varepsilon \sin k_{w} x+\frac{1}{2 !} \varphi_{y y}(x, a) \varepsilon^{2} \sin ^{2} k_{w} x+\ldots . .-$ $-\varphi(x, b)-\varphi_{y}(x, b) \varepsilon \sin k_{w} x-\frac{1}{2 !} \varphi_{y y}(x, b) \varepsilon^{2} \sin ^{2} k_{w} x-\ldots \ldots$

Now substituting (2.1) into (1.1,1.2 and 2.2) and equating coefficients of like powers of $\varepsilon$, we have order $\varepsilon^{0}$

$$
\begin{aligned}
& \varphi_{0 x x}+\varphi_{0 y y}+w^{2} \varphi_{0}=0 \\
& \varphi_{0 y}(x, a)-\varphi_{0 y}(x, b)=0 \\
& \varphi_{0}(x, a)-\varphi_{0}(x, b)=0
\end{aligned}
$$

order $\varepsilon^{1}$

$$
\begin{aligned}
& \varphi_{1 x x}+\varphi_{1 y y}+w^{2} \varphi_{1}=0 \\
& \varphi_{1 y}(x, a)-\varphi_{1 y}(x, b)=0 \\
& \varphi_{1}(x, a)-\varphi_{1}(x, b)=-\varphi_{0 y}(x, a) \sin k_{w} x+\varphi_{0 y}(x, b) \sin k_{w} x
\end{aligned}
$$

\section{II - Applying method of separation of variables}

Since the problem (2.3-2.5) is homogenous and with constant coefficients, therefore can be solved by separation of variables as the following

$$
\begin{aligned}
& \text { Let } \varphi_{0}(x, y)=X(x) Y(y) \\
& X^{\prime \prime} Y+X Y^{\prime \prime}+w^{2} X Y=0 \\
& \varphi_{0 y}(x, a)-\varphi_{0 y}(x, b)=0 \quad \Rightarrow Y^{\prime}(a)-Y^{\prime}(b)=0 \\
& \varphi_{0}(x, a)-\varphi_{0}(x, b)=0 \Rightarrow Y(a)-Y(b)=0
\end{aligned}
$$

Dividing (2.10) by $X Y$ and equating to the separation constants, we can write 
$\frac{-X^{\prime \prime}}{X}=\frac{Y^{\prime \prime}}{Y}+w^{2}=c, c>0$

or

$X^{\prime \prime}+k^{2} X=0 \quad, C=k^{2}$

$Y^{\prime \prime}+\left(w^{2}-k^{2}\right) Y=0$

Both equations $(2.13,2.14)$ have solution

$X=\exp (\mp i k x)$

$Y=c_{1} \sin \sqrt{w^{2}-k} y+c_{2} \cos \sqrt{w^{2}-k} y \quad,\left(c_{1}, c_{2}\right.$ arbitrary $)$

Substituting (2.16) in $(2.11,2.12)$, and we find the determinant of the coefficients and equating it to zero, we have

$\cos \sqrt{w^{2}-k^{2}}(b-a)=1 \quad, \sqrt{w^{2}-k^{2}}(b-a)=2 n \pi$,

$k_{n}^{2}=w^{2}-\left(\frac{2 n \pi}{b-a}\right)^{2}, n=1,2,3, \ldots \ldots$

and hence

$\varphi_{0}=\exp \left(i k_{n} x\right)\left(c_{1} \sin \left(\frac{2 n \pi}{b-a}\right) y+c_{2} \cos \left(\frac{2 n \pi}{b-a}\right) y\right)$

Substituting (2.18) into (2.8), we have

$\varphi_{1}(x, a)-\varphi_{1}(x, b)=\delta_{1} \exp \left(i\left(k_{n}+k_{w}\right)\right) x+\delta_{2} \exp \left(i\left(k_{n}-k_{w}\right)\right) x$

where

$$
\left.\begin{array}{l}
\delta_{1}=\frac{1}{2 i}\left(\frac{2 n \pi}{b-a}\right)\left(-\left(\cos \left(\frac{2 n \pi}{b-a}\right) a-\cos \left(\frac{2 n \pi}{b-a}\right) b\right)+\left(\sin \left(\frac{2 n \pi}{b-a}\right) a-\sin \left(\frac{2 n \pi}{b-a}\right) b\right)\right. \\
\delta_{2}=\frac{1}{2 i}\left(\frac{2 n \pi}{b-a}\right)\left(\left(\cos \left(\frac{2 n \pi}{b-a}\right) a-\cos \left(\frac{2 n \pi}{b-a}\right) b\right)-\left(\sin \left(\frac{2 n \pi}{b-a}\right) a-\sin \left(\frac{2 n \pi}{b-a}\right) b\right)\right.
\end{array}\right\}
$$

To find the solution of $\left(2.6,2.7\right.$ and 2.19) for $\varphi_{1}$, we note that the boundary conditions (2.19) is nonhomogenous, then the variables be separated as follows

$$
\varphi_{1}=\phi_{1}(y) \exp \left(i\left(k_{n}+k_{w}\right)\right) x+\phi_{2}(y) \exp \left(i\left(k_{n}-k_{w}\right)\right) x
$$

Put (2.21) in (2.6, 2.7 and 2.19), we have

$\left(\phi_{1}^{\prime \prime}(y)+\alpha_{1}^{2} \phi_{1}(y)\right) \exp \left(i\left(k_{n}+k_{w}\right)\right) x+\left(\phi_{2}^{\prime \prime}(y)+\alpha_{2}^{2} \phi_{2}(y)\right) \exp \left(i\left(k_{n}-k_{w}\right)\right) x=0$

where

$\alpha_{1}^{2}=w^{2}-\left(k_{n}+k_{w}\right)^{2}, \alpha_{2}^{2}=w^{2}-\left(k_{n}-k_{w}\right)^{2}$

$\left(\phi_{1}^{\prime}(a)-\phi_{1}^{\prime}(b)\right) \exp \left(i\left(k_{n}+k_{w}\right)\right) x+\left(\phi_{2}^{\prime}(a)-\phi_{2}^{\prime}(b)\right) \exp \left(i\left(k_{n}-k_{w}\right)\right) x=0$

$\phi_{1}(a) \exp \left(i\left(k_{n}+k_{w}\right)\right) x+\phi_{2}(a) \exp \left(i\left(k_{n}-k_{w}\right)\right) x-\phi_{1}(b) \exp \left(i\left(k_{n}+k_{w}\right)\right) x-$

$-\phi_{2}(b) \exp \left(i\left(k_{n}-k_{w}\right)\right) x=\delta_{1} \exp \left(i\left(k_{n}+k_{w}\right)\right) x+\delta_{2} \exp \left(i\left(k_{n}-k_{w}\right)\right) x$ 
Equating the coefficients of each of exponentials on both sides, we obtain

$$
\begin{aligned}
& \phi_{1}^{\prime \prime}(y)+\alpha_{1}^{2} \phi_{1}(y)=0, \phi_{1}^{\prime}(a)-\phi_{1}^{\prime}(b)=0, \phi_{1}(a)-\phi_{1}(b)=\delta_{1} \\
& \phi_{2}^{\prime \prime}(y)+\alpha_{2}^{2} \phi_{2}(y)=0, \phi_{2}^{\prime}(a)-\phi_{2}^{\prime}(b)=0, \phi_{2}(a)-\phi_{2}(b)=\delta_{2}
\end{aligned}
$$

The general solution of (2.23) is

$\phi_{1}=A_{1} \cos \alpha_{1} y+A_{2} \sin \alpha_{1} y$, and substituting in boundary conditions we have

$A_{1}\left(-\alpha_{1} \sin \alpha_{1} a+\alpha_{1} \sin \alpha_{1} b\right)+A_{2}\left(\alpha_{1} \cos \alpha_{1} a-\alpha_{1} \cos \alpha_{1} b\right)=0$

$A_{1}\left(\cos \alpha_{1} a-\cos \alpha_{1} b\right)+A_{2}\left(\sin \alpha_{1} a-\sin \alpha_{1} b\right)=\delta_{1}$

hence

$$
\phi_{1}=\frac{\delta_{1}\left(\cos \alpha_{1} a-\cos \alpha_{1} b\right)}{2\left(1-\cos \alpha_{1}(b-a)\right)} \cos \alpha_{1} y+\frac{\delta_{1}\left(\sin \alpha_{1} a-\sin \alpha_{1} b\right)}{2\left(1-\cos \alpha_{1}(b-a)\right)} \sin \alpha_{1} y
$$

Similarly the solution of (2.24) is

$$
\phi_{2}=\frac{\delta_{2}\left(\cos \alpha_{2} a-\cos \alpha_{2} b\right)}{2\left(1-\cos \alpha_{2}(b-a)\right)} \cos \alpha_{2} y+\frac{\delta_{2}\left(\sin \alpha_{2} a-\sin \alpha_{2} b\right)}{2\left(1-\cos \alpha_{2}(b-a)\right)} \sin \alpha_{2} y
$$

Therefore from (2.21), we have

$$
\begin{aligned}
\varphi_{1} & =\left(\frac{\delta_{1}\left(\cos \alpha_{1} a-\cos \alpha_{1} b\right)}{2\left(1-\cos \alpha_{1}(b-a)\right)} \cos \alpha_{1} y+\right. \\
& \left.+\frac{\delta_{1}\left(\sin \alpha_{1} a-\sin \alpha_{1} b\right)}{2\left(1-\cos \alpha_{1}(b-a)\right)} \sin \alpha_{1} y\right) \exp \left(i\left(k_{n}+k_{w}\right)\right) x+ \\
& +\left(\frac{\delta_{2}\left(\cos \alpha_{2} a-\cos \alpha_{2} b\right)}{2\left(1-\cos \alpha_{2}(b-a)\right)} \cos \alpha_{2} y+\right. \\
& \left.+\frac{\delta_{2}\left(\sin \alpha_{2} a-\sin \alpha_{2} b\right)}{2\left(1-\cos \alpha_{2}(b-a)\right)} \sin \alpha_{2} y\right) \exp \left(i\left(k_{n}-k_{w}\right)\right) x
\end{aligned}
$$

Substituting $(2.18,2.25)$ into $(2.1)$, we obtain

$$
\begin{aligned}
& \varphi=\exp (i k x)\left(\sin \frac{2 n \pi}{b-a} y+\cos \frac{2 n \pi}{b-a} y\right)+\varepsilon\left(\left(\frac{\delta_{1}\left(\cos \alpha_{1} a-\cos \alpha_{1} b\right)}{2\left(1-\cos \alpha_{1}(b-a)\right)} \cos \alpha_{1} y+\right.\right. \\
& \left.+\frac{\delta_{1}\left(\sin \alpha_{1} a-\sin \alpha_{1} b\right)}{2\left(1-\cos \alpha_{1}(b-a)\right)} \sin \alpha_{1} y\right) \exp \left(i\left(k_{n}+k_{w}\right)\right) x+ \\
& +\left(\frac{\delta_{2}\left(\cos \alpha_{2} a-\cos \alpha_{2} b\right)}{2\left(1-\cos \alpha_{2}(b-a)\right)} \cos \alpha_{2} y+\right. \\
& \left.\left.+\frac{\delta_{2}\left(\sin \alpha_{2} a-\sin \alpha_{2} b\right)}{2\left(1-\cos \alpha_{2}(b-a)\right)} \sin \alpha_{2} y\right) \exp \left(i\left(k_{n}-k_{w}\right)\right) x\right)+\ldots \ldots .
\end{aligned}
$$

If either $\cos \alpha_{1}(b-a)=1 \quad$ or $\quad \cos \alpha_{2}(b-a)=1$, the second term tends to infinity, and therefore the series (2.26) is nonuniform. 


$$
\begin{array}{r}
\text { Since } \cos \alpha_{1}(b-a)=1 \Rightarrow \alpha_{1}=\frac{2 m \pi}{b-a}, m=0,1,2, \ldots \ldots . \\
w^{2}-\left(k_{n}+k_{w}\right)^{2} \approx\left(\frac{2 m \pi}{b-a}\right)^{2} \text { or } \quad w^{2}-\left(k_{n}-k_{w}\right)^{2} \approx\left(\frac{2 m \pi}{b-a}\right)^{2}
\end{array}
$$

But $\quad w^{2}-\left(\frac{2 m \pi}{b-a}\right)^{2}=k_{m}^{2}$ from (2.17), hence (2.27) can be written as $\left(k_{n}+k_{w}\right)^{2} \approx k_{m}^{2} \quad$ or $\quad\left(k_{n}-k_{w}\right)^{2} \approx k_{m}^{2} \quad$ or $\quad k_{w}=\mp k_{n} \mp k_{m}$

To determine an expansion valid when $k_{w} \approx k_{n}-k_{m}$, let the parameter $\sigma$ as $\quad k_{w}=k_{n}-k_{m}+\varepsilon \sigma$

\section{III - Using method of multiple scales}

Using the method of multiple scales in [5] and seek the expansion in the form

$$
\varphi(x, y, \varepsilon)=\varphi\left(x_{0}, x_{1}, y, \varepsilon\right)=\varphi_{0}\left(x_{0}, x_{1}, y\right)+\varepsilon \varphi_{1}\left(x_{0}, x_{1}, y\right)+\ldots \ldots
$$

where $x_{0}=x$ and $x_{1}=\varepsilon x$, thus

$$
\left.\begin{array}{l}
\frac{\partial}{\partial x}=\frac{\partial}{\partial x_{0}}+\varepsilon \frac{\partial}{\partial x_{1}}+\ldots \ldots \\
\frac{\partial^{2}}{\partial x^{2}}=\frac{\partial^{2}}{\partial x_{0}^{2}}+2 \varepsilon \frac{\partial^{2}}{\partial x_{0} \partial x_{1}}+\ldots . .
\end{array}\right\}
$$

Substituting $(2.28,2.29)$ in $(1.1,1.2$ and 2.2$)$ and equating coefficients of likes power of $\varepsilon$, we obtain order $\varepsilon^{0}$

$$
\begin{aligned}
& \varphi_{0 x_{0} x_{0}}+\varphi_{0 y y}+w^{2} \varphi_{0}=0 \\
& \varphi_{0 y}\left(x_{0}, x_{1}, a\right)-\varphi_{0 y}\left(x_{0}, x_{1}, b\right)=0 \\
& \varphi_{0}\left(x_{0}, x_{1}, a\right)-\varphi_{0}\left(x_{0}, x_{1}, b\right)=0
\end{aligned}
$$

order $\varepsilon^{1}$

$$
\begin{aligned}
& \varphi_{1 x_{0} x_{0}}+\varphi_{1 y y}+w^{2} \varphi_{1}=-2 \varphi_{0 x_{0} x_{1}} \\
& \varphi_{1 y}\left(x_{0}, x_{1}, a\right)-\varphi_{1 y}\left(x_{0}, x_{1}, b\right)=0 \\
& \varphi_{1}\left(x_{0}, x_{1}, a\right)-\varphi_{1}\left(x_{0}, x_{1}, b\right)=-\varphi_{0 y}\left(x_{0}, x, a\right) \sin k_{w} x_{0}+\varphi_{0 y}\left(x_{0}, x, b\right) \sin k_{w} x_{0}
\end{aligned}
$$

The solution of (2.30) can be obtained by separating variables, however instead of making $\varphi_{0}$ contain only one mode, we make $\varphi_{0}$ contain the two modes, the mth and nth mode and hence can be written as

$$
\begin{aligned}
\varphi_{0}= & A_{n}\left(x_{1}\right)\left(\cos \frac{2 n \pi}{b-a} y+\sin \frac{2 n \pi}{b-a} y\right) \exp \left(i k_{n} x_{0}\right)+ \\
& +A_{m}\left(x_{1}\right)\left(\cos \frac{2 m \pi}{b-a} y+\sin \frac{2 m \pi}{b-a} y\right) \exp \left(i k_{m} x_{0}\right)
\end{aligned}
$$


where $k_{n}, k_{m}$ are defined by (2.17) and $A_{n}, A_{m}$ will be determined.

Substituting (2.34) into (2.31, 2.33), we have

$$
\begin{aligned}
& \frac{\partial^{2} \varphi_{1}}{\partial x_{0}^{2}}+\frac{\partial^{2} \varphi_{1}}{\partial y^{2}}+w^{2} \varphi_{1}=-2 i k_{n} A_{n}^{\prime}\left(x_{1}\right)\left(\cos \frac{2 n \pi}{b-a} y+\sin \frac{2 n \pi}{b-a} y\right) \exp \left(i k_{n} x_{0}\right)- \\
& -2 i k_{m} A_{m}^{\prime}\left(x_{1}\right)\left(\cos \frac{2 m \pi}{b-a} y+\sin \frac{2 m \pi}{b-a} y\right) \exp \left(i k_{m} x_{0}\right) \\
& \varphi_{1}\left(x_{0}, x_{1}, a\right)-\varphi_{1}\left(x_{0}, x_{1}, b\right)=-A_{n}\left(x_{1}\right)\left(\frac{2 n \pi}{b-a}\right)\left(-\sin \frac{2 n \pi}{b-a} a+\right. \\
& \left.+\cos \frac{2 n \pi}{b-a} a\right) \sin \left(k_{w} x_{0}\right) \exp \left(i k_{n} x_{0}\right)-A_{m}\left(x_{1}\right)\left(\frac{2 m \pi}{b-a}\right)\left(-\sin \frac{2 m \pi}{b-a} a+\right. \\
& \left.+\cos \frac{2 m \pi}{b-a} a\right) \sin \left(k_{w} x_{0}\right) \exp \left(i k_{m} x_{0}\right)+A_{n}\left(x_{1}\right)\left(\frac{2 n \pi}{b-a}\right)\left(-\sin \frac{2 n \pi}{b-a} b+\right. \\
& \left.+\cos \frac{2 n \pi}{b-a} b\right) \sin \left(k_{w} x_{0}\right) \exp \left(i k_{n} x_{0}\right)+A_{m}\left(x_{1}\right)\left(\frac{2 m \pi}{b-a}\right)\left(-\sin \frac{2 m \pi}{b-a} b+\right. \\
& \left.+\cos \frac{2 m \pi}{b-a} b\right) \sin \left(k_{w} x_{0}\right) \exp \left(i k_{m} x_{0}\right) \\
& \varphi_{1}\left(x_{0}, x_{1}, a\right)-\varphi_{1}\left(x_{0}, x_{1}, b\right)=\delta_{1 n} A_{n} \exp \left(i\left(k_{n}+k_{w}\right)\right) x_{0}+ \\
& \quad+\delta_{2 n} A_{n} \exp \left(i\left(k_{n}-k_{w}\right)\right) x_{0}+ \\
& \quad+\delta_{1 m} A_{m} \exp \left(i\left(k_{m}+k_{w}\right)\right) x_{0}+\delta_{2 m} A_{m} \exp \left(i\left(k_{m}-k_{w}\right)\right) x_{0}
\end{aligned}
$$

where $\delta_{1 n}, \delta_{2 n}$ are defined by (2.20) and $\delta_{1 m}, \delta_{2 m}$ are also defined by (2.20) if $n$ is replaced by $\mathrm{m}$.

To determine the solvability conditions for the first problem, substitute $k_{w}=k_{n}-k_{m}+\varepsilon \sigma$ into (2.36), we have

$$
\begin{aligned}
& \varphi_{1}\left(x_{0}, x_{1}, a\right)-\varphi_{1}\left(x_{0}, x_{1}, b\right)=\delta_{1 n} A_{n} \exp \left(i\left(2 k_{n}-k_{m}+\varepsilon \sigma\right)\right) x_{0}+ \\
& \quad+\delta_{2 n} A_{n} \exp \left(i\left(k_{m}-\varepsilon \sigma\right)\right) x_{0}+\delta_{1 m} A_{m} \exp \left(i\left(k_{n}+\varepsilon \sigma\right)\right) x_{0}+ \\
& \quad+\delta_{2 m} A_{m} \exp \left(i\left(2 k_{m}-k_{n}-\varepsilon \sigma\right)\right) x_{0} \\
& =\delta_{1 n} A_{n} \exp \left(i \sigma x_{1}\right) \exp \left(i\left(2 k_{n}-k_{m}\right)\right) x_{0}+\delta_{2 n} A_{n} \exp \left(-i \sigma x_{1}\right) \exp \left(i k_{m} x_{0}\right)+ \\
& +\delta_{1 m} A_{m} \exp \left(i k_{n} x_{0}\right) \exp \left(i \sigma x_{1}\right)+\delta_{2 m} A_{m} \exp \left(-i \sigma x_{1}\right) \exp \left(i\left(2 k_{m}-k_{n}\right)\right) x_{0}
\end{aligned}
$$

where $x_{1}=\varepsilon x_{0}$

We note that the terms $\exp \left(i k_{m} x_{0}\right)$ and $\exp \left(i k_{n} x_{0}\right)$ in (2.35) and (2.37) may lead to incompatibilities and solvability conditions must be imposed on them. These solvability conditions can be obtained by seeking a particular solution corresponding to these terms in the form 
$\varphi_{1}=\phi_{n}\left(x_{1}, y\right) \exp \left(i k_{n} x_{0}\right)+\phi_{m}\left(x_{1}, y\right) \exp \left(i k_{m} x_{0}\right)$

Substituting (2.38) into (2.32, 2.35 and 2.37) and equating the coefficients of $\exp \left(i k_{n} x_{0}\right)$ and $\exp \left(i k_{m} x_{0}\right)$ on both sides, we have

$\left.\begin{array}{l}\frac{\partial^{2} \phi_{n}}{\partial y^{2}}+\left(\frac{2 n \pi}{b-a}\right)^{2} \phi_{n}=-2 i k_{n} A_{n}^{\prime}\left(\cos \frac{2 n \pi}{b-a} y+\sin \frac{2 n \pi}{b-a} y\right) \\ \phi_{n y}\left(x_{1}, a\right)-\phi_{n y}\left(x_{1}, b\right)=0 \\ \phi_{n}\left(x_{1}, a\right)-\phi_{n}\left(x_{1}, b\right)=\delta_{1 m} A_{m} \exp \left(i \sigma x_{1}\right)\end{array}\right\}$

$\frac{\partial^{2} \phi_{m}}{\partial y^{2}}+\left(\frac{2 m \pi}{b-a}\right)^{2} \phi_{m}=-2 i k_{m} A_{m}^{\prime}\left(\cos \frac{2 m \pi}{b-a} y+\sin \frac{2 m \pi}{b-a} y\right)$

$\phi_{m y}\left(x_{1}, a\right)-\phi_{m y}\left(x_{1}, b\right)=0$

$\phi_{m}\left(x_{1}, a\right)-\phi_{m}\left(x_{1}, b\right)=\delta_{2 n} A_{n} \exp \left(-i \sigma x_{1}\right)$

Thus, determining the solvability conditions $\phi_{1}$ has been transformed into determining the solvability conditions for $\phi_{n}$ and $\phi_{m}$.

We note that the equation in (2.39) is self - adjoint, the solution of the adjoint problem can be taken as $u=\cos \frac{2 n \pi}{b-a} y+\sin \frac{2 n \pi}{b-a} y$

Multiplying the equation in (2.39) by $u(y)$ and integrating the result by parts from $y=a$ to $y=b$ to transfer the derivatives from $\phi_{n}$ to $u$, we obtain

$$
\begin{aligned}
& \int_{a}^{b} u\left(\frac{\partial^{2} \phi_{n}}{\partial y^{2}}+\left(\frac{2 n \pi}{b-a}\right)^{2} \phi_{n}\right) d y=\int_{a}^{b}\left(-2 i k_{n} A_{n}^{\prime}\left(\cos \frac{2 n \pi}{b-a} y+\sin \frac{2 n \pi}{b-a} y\right)^{2}\right) d y \\
& \int_{a}^{b}\left(u^{\prime \prime}+\left(\frac{2 n \pi}{b-a}\right)^{2} u\right) \phi_{n} d y+\left|\frac{\partial \phi_{n}}{\partial y} u-\phi_{n} u^{\prime}\right|_{a}^{b}=-2 i k_{n} A_{n}^{\prime}(b-a)
\end{aligned}
$$

To find the boundary conditions for adjoint problem, put the right side in (2.41) equal to zero and using the homogenous boundary conditions in (2.39), we obtain

$$
\frac{\partial \phi_{n}(b)}{\partial y}(u(b)-u(a))-\phi_{n}(b)\left(u^{\prime}(b)-u^{\prime}(a)\right)=0
$$

Equating the coefficients of $\frac{\partial \phi_{n}(b)}{\partial y}$ and $\phi_{n}(b)$ by zero, we have

$$
u(b)=u(a), u^{\prime}(b)=u^{\prime}(a)
$$

which are the boundary conditions for adjoint problem. 
Now from (2.41) and using the nonhomogenous conditions in (2.39) and boundary conditions (2.42), we obtain

$-\left.\phi_{n}(x) u^{\prime}(x)\right|_{a} ^{b}=-2 i k_{n} A_{n}^{\prime}(b-a)$

$-\left(\phi_{n}(b) u^{\prime}(b)-\phi_{n}(a) u^{\prime}(a)\right)=-2 i k_{n} A_{n}^{\prime}(b-a)$

or

$\delta_{1 m} A_{m} \exp \left(i \sigma x_{1}\right)\left(-\sin \frac{2 n \pi}{b-a} b+\cos \frac{2 n \pi}{b-a} b\right) \frac{2 n \pi}{b-a}=-2 i k_{n} A_{n}^{\prime}(b-a)$

therefore

$$
A_{n}^{\prime}=\left(\delta_{1 m} A_{m} \exp \left(i \sigma x_{1}\right)\left(\cos \frac{2 n \pi}{b-a} b-\sin \frac{2 n \pi}{b-a} b\right) \frac{2 n \pi}{b-a}\right) \frac{i k_{n}^{-1}}{2(b-a)}
$$

and this is the solvability condition for problem (2.39).

Similarly if $m \neq 0$, the solvability condition for problem (2.40)

$A_{m}^{\prime}=\left(\delta_{2 n} A_{n} \exp \left(i \sigma x_{1}\right)\left(\cos \frac{2 m \pi}{b-a} b-\sin \frac{2 m \pi}{b-a} b\right) \frac{2 m \pi}{b-a}\right) \frac{i k_{m}^{-1}}{2(b-a)}$

If we let $A_{n}=a_{n} \exp \left(i \gamma_{1} x_{1}\right), A_{m}=a_{m} \exp \left(i \gamma_{2} x_{1}\right)$

where $a_{n}, a_{m}, \gamma_{1}$ and $\gamma_{2}$ are constants, then it follows from (2.43) and (2.44)

$$
\begin{aligned}
& i \gamma_{1} a_{n}=\left(\delta_{1 m} a_{m}\left(\cos \frac{2 n \pi}{b-a} b-\sin \frac{2 n \pi}{b-a} b\right) \frac{2 n \pi}{b-a}\right) \frac{i k_{n}^{-1}}{2(b-a)} \\
& i \gamma_{2} a_{m}=\left(\delta_{2 n} a_{n}\left(\cos \frac{2 m \pi}{b-a} b-\sin \frac{2 m \pi}{b-a} b\right) \frac{2 m \pi}{b-a}\right) \frac{i k_{m}^{-1}}{2(b-a)} \\
& \gamma_{2}=\gamma_{1}-\sigma
\end{aligned}
$$

Eliminating $\gamma_{2}$ and $a_{m}$ from (2.46) by using (2.48), we have

$$
\begin{gathered}
\gamma_{1}\left(\gamma_{1}-\sigma\right)=\left(\delta_{1 m} \delta_{2 n}\left(\cos \frac{2 n \pi}{b-a} b-\sin \frac{2 n \pi}{b-a} b\right)\left(\cos \frac{2 m \pi}{b-a} b-\sin \frac{2 m \pi}{b-a} b\right)\right. \\
\left(\frac{2 n \pi}{b-a}\right)\left(\frac{2 m \pi}{b-a}\right)\left(\frac{k_{n}^{-1}}{2(b-a)}\right)\left(\frac{k_{m}^{-1}}{2(b-a)}\right)
\end{gathered}
$$

or

$$
\begin{gathered}
\gamma_{1}^{2}-\sigma \gamma_{1}-\left(\delta_{1 m} \delta_{2 n}\left(\cos \frac{2 n \pi}{b-a} b-\sin \frac{2 n \pi}{b-a} b\right)\left(\cos \frac{2 m \pi}{b-a} b-\sin \frac{2 m \pi}{b-a} b\right)\right. \\
\left(\frac{2 n \pi}{b-a}\right)\left(\frac{2 m \pi}{b-a}\right)\left(\frac{k_{n}^{-1} k_{m}^{-1}}{4(b-a)^{2}}\right)=0
\end{gathered}
$$




$$
\begin{aligned}
\gamma_{1}= & \frac{1}{2} \sigma \mp \frac{1}{2}\left(\sigma^{2}+\delta_{1 m} \delta_{2 n}\left(\cos \frac{2 n \pi}{b-a} b-\sin \frac{2 n \pi}{b-a} b\right)\left(\cos \frac{2 m \pi}{b-a} b-\sin \frac{2 m \pi}{b-a} b\right)\right. \\
& \left.\left(\frac{2 n \pi}{b-a}\right)\left(\frac{2 m \pi}{b-a}\right)\left(\frac{k_{n}^{-1} k_{m}^{-1}}{b-a}\right)\right)^{\frac{1}{2}}
\end{aligned}
$$

When substitute the value of $\gamma_{1}$ into (2.45), we find the values of $A_{n}, A_{m}$ and then substitute in (2.43), (2.44), we obtain the solvability conditions of the problems (2.39), (2.40).

\section{Conclusions}

The perturbation method has been applied to find solvability conditions of the $2^{\text {nd }}$ order boundary value problem (1.1-1.3) which are

$$
\begin{aligned}
& A_{n}^{\prime}=\left(\delta_{1 m} A_{m} \exp \left(i \sigma x_{1}\right)\left(\cos \frac{2 n \pi}{b-a} b-\sin \frac{2 n \pi}{b-a} b\right) \frac{2 n \pi}{b-a}\right) \frac{i k_{n}^{-1}}{2(b-a)} \\
& A_{m}^{\prime}=\left(\delta_{2 n} A_{n} \exp \left(i \sigma x_{1}\right)\left(\cos \frac{2 m \pi}{b-a} b-\sin \frac{2 m \pi}{b-a} b\right) \frac{2 m \pi}{b-a}\right) \frac{i k_{m}^{-1}}{2(b-a)}
\end{aligned}
$$

These conditions were satisfied.

\section{References}

() محمود، لكرم عسان وجلم، إسبراء عبد العالي.(2006):"شروط الطل لمسألة قيم

حدوية خاصة بطريقة التثويش".مجلة التربية والعلم، مجل ـد (18)، الع دد (2)،

$$
\text { ص(103-90). }
$$

2) Chikwendu, S. C. (1982): "Fourier transform perturbation solution of elliptic equations with small nonlinearity". Quarterly of applied mathematics, October.

3) Cimatti, G. (1986): "Perturbation technique in the theory of lubrication". Quarterly of applied mathematics,Vol. (XII), No.(1), p (97-108).

4) Mahmood, A. H. (2000): "Solvability conditions for certain eigenvalue problem by perturbation technique". J. of Educ. and Sci.Vol.(33), university of mosul, Mosul, Iraq.

5) Nayfeh, A. H. (1973): "Perturbation methods". WilegInterscience publication, New York.

6) Nayfeh, A. H. (1981): "Introduction to perturbation methods". John Wiley and Sons, New York.

7) Noor, M. A. and Mohyud-Din, S. T. (2007): "Perturbation method for solving fourth order boundary value problems". Hindawi publishing corporation, Mathematical problem in engineering. Vol. (2007). 